\title{
PYODERMA GANGRENOSUM COMPLICATING A SKIN GRAFT FOR BURNS
}

\author{
Sushma Chowdary Kosaraju1, Pratap Duggirala²
}

\section{HOW TO CITE THIS ARTICLE:}

Sushma Chowdary Kosaraju, Pratap Duggirala. "Pyoderma Gangrenosum Complicating a Skin Graft for Burns". Journal of Evolution of Medical and Dental Sciences 2015; Vol. 4, Issue 37, May 07; Page: 6537-6541,

DOI: $10.14260 /$ jemds/2015/947

ABSTRACT: This is a rare presentation of pyoderma gangrenosum complicating a skin graft for burns.

KEYWORDS: Pyoderma gangrenosum, burns, split thickness skin graft.

INTRODUCTION: Pyoderma gangrenosum (PG) is a rare, non-infective neutrophilic dermatosis commonly associated with underlying systemic disease (Most often inflammatory bowel disease, haematological disease or arthritis) in about $50-70 \%$ of patients. Pathergy phenomenon occurs in up to $50 \%$ of PG patients, whereby new lesions can be induced at sites of minor skin trauma including venepucture, vaccination and surgical procedures.[1][2] Diagnosis is based on typical clinical features and exclusion of other cutaneous ulcerating diseases.[3]

CASE REPORT: An 18 year old female was admitted in our hospital in the plastic surgery unit with $15 \%$ total body surface area - post burn raw area (PBRA) [Figure 1]. She gave a history of attempted suicide with kerosene with $2^{\text {nd }} / 3^{\text {rd }}$ degree burns, four months back, on her lower face, neck, upper chest involving both the breasts, arms and left thigh for which she was treated conservatively with daily dressings in another hospital.

At the time of presentation, it was noted that PBRA wound was covered with slough and initially silver sulfadiazine and saline soaked dressings were applied along with appropriate injectable antibiotics. Ten days later, she developed few haemorrhagic bullae over the raw area [Figure 2]. The bullae were drained under aseptic conditions. She was planned for three stages of sequential surgical debridements and grafting. In the first stage, grafting was performed to her neck area with a thick split thickness skin graft (STSG) taken from right thigh. In the second and third stages, thin to intermediate STSG was performed for trunk and arms, the donor area being the thighs and the legs [Figure 3,4]. The postoperative dressings were removed on the second day of surgery, followed by daily dressings for all the three stages. Postoperatively, the cultures of swabs taken were negative for bacteria.

The initial graft has taken well in the neck region, but over the trunk and arms there was loss of graft taken-up at half of the area. The donor area healed well except for a small area over the right thigh where the thick STSG was harvested.

For personal reasons, the patient got discharged against medical advice. Three weeks after the third stage of grafting, at home she noticed multiple small painful breakdowns over the graft accepted and lost areas of the trunk, arms and the donor site of right thigh. These breakdowns progressively enlarged and coalesced.

She presented to our plastic surgery unit two months after discharge with multiple, painful, non-healing ulcers over previous grafted areas and so was re-admitted. The ulcerations became larger, despite daily dressings and systemic antibiotics. She had no systemic symptoms except for 
mild fever. Her general and systemic examination was normal except for anemia. Other laboratory parameters were within normal limits. Culture tests from ulcers were sterile.

The patient was referred to Dermatology OPD \& at the time of examination, there were multiple, raised, painful granulating ulcers with necrotic slough over trunk, arms and right thigh. [Figure 5]. The differential diagnosis of pyoderma gangrenosum and bullous pemphigoid were considered. Ulcer biopsy showed dense predominantly neutrophilic infiltrate on a background of proliferating granulation tissue involving most of the dermis [Figure $6 \mathrm{a}, \mathrm{b}$ ]. Overlying epidermis is deficient \& surface was covered by fibrin, necrotic cells \& plasma. These findings are suggestive of PG. Direct immunoflourescence done on perilesional skin was negative. Patient later underwent colonoscopy which showed no abnormality. Serum and urinary protein electrophoresis were normal.

Treatment was commenced with oral cyclosporine $(3 \mathrm{mg} / \mathrm{kg} / \mathrm{d})$ and daily dressings with topical fusidic acid and betamethasone valerate. This resulted in clinical improvement within 4 days. The areas of ulceration ceased to enlarge and began to re-epithelialize, within the first week [Figure 7] and almost healed completely by four weeks [Figure 8]. Cyclosporine was tapered slowly after 4 weeks and discontinued after six weeks. She is under regular follow up with no recurrence of ulcerations since ten months.

DISCUSSION: Post-surgical pyoderma gangrenosum (PSPG), also known as Post-operative gangrene of Cullen, is a rare complication of surgical procedures described by Cullen for the first time in 1924.[4] The symptoms of PSPG begin between few days to sometimes six weeks postoperatively. This can affect any incision site, however, the abdominal wall and the breasts are the most affected areas. The nipple areolar complex is spared, which is highly suggestive, if not pathognomonic of PSPG.[5]

In the above reported case, symptoms of PG began three weeks after the third stage of skin grafting, with involvement of trunk except the nipple areolar complex, arms and a part of right thigh. There is sparing of the grafted neck and donor areas. A dramatic response to oral cyclosporine therapy was observed. An additional unusual feature in our patient was the lack of an associated systemic disease, but long term surveillance is often indicated as PG can also pre-date the associated conditions by up to 10 years.[6]

Pyoderma gangrenosum (PG) in burns after skin grafting is a rare presentation, with very few cases reported in the literature. Kalu PU et al, [6] reported a case of PG after a scald in 2007. C.C. West et al, ${ }^{[7]}$ in 2010 reported three cases of PG in burns. To our knowledge only two case reports of PG following the skin grafting operation for a non-healing traumatic ulcer were described in literature, first by Klevanski.[8] in 1958 \& second by P. Betto.[9] in 2010.

In our patient, it is still not clear why the hemorrhagic bullae developed over the raw area four months after the initial burn injury and the reason for graft not being taken in half the area in the second and third stages of grafting cannot be explained. Biopsy and histopathological examination at the time of development of haemorrhagic bullae or at the time of graft not being taken might have cleared whether PG presented secondary to burn injury or due to debridement and grafting.

Early diagnoses of $\mathrm{PG}$ is essential to prevent pathergy. If presented to clinical team unfamiliar with PG, it may be misdiagnosed as infection or treated with surgical debridement, which is potentiality dangerous. 


\section{CASE REPORT}

\section{REFERENCES:}

1. Jackson JM, Callen JP. Pyoderma gangrenosum: an expert commentary. Expert Rev Dermatol 2006; 1: 391-400.

2. Wollina U. Pyoderma gangrenosum- a review. Orphanet J Rare Dis 2007; 2: 19.

3. Callen JP, Jackson JM. Pyoderma gangrenosum: an update. Rheum Dis Clin N Am 2007; 33: 787802.

4. Cullen TS. A progressively enlarging ulcer of abdominal wall involving skin and fat, following drainage of an abdominal abscess apparently from appendiceal origin. Surg Gynecol Obstet 1924; 38: 579-82.

5. Ouazzani A, Berthe J-V, De Fountaine S. Post-surgical pyoderma gangrenosum: a clinical entity. Acta Chir Belg 2007; 107: 424-8.

6. Kalu PU, Williams G. Scalding as an unusual cause of pyoderma gangrenosum. Burns 2007; 33: 105-8.

7. West CC, Morritt AN, Ralston DR. Pyoderma gangrenosum in burns: a report of 3 cases and review of the literature. Burns 2010; 36: e56-9.

8. Klevansky H, Macrae RC. Pyoderma gangrenosum complicating a simple skin graft. S Afr Med J 1958; 32: 964-5.

9. Betto P, Germi L, Panigalli S, Veller-Fornasa C. Atypical Pyoderma gangrenosum complicating a skin graft. G Ital Dermatol Venereol 2010; 145: 317-318.

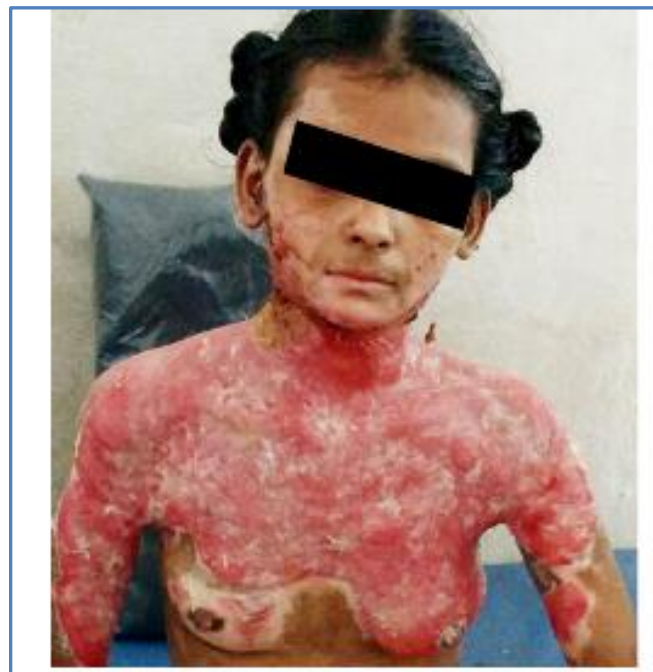

Figure 1: Post Burn Raw Area (PBRA) involving neck, proximal arms and trunk.

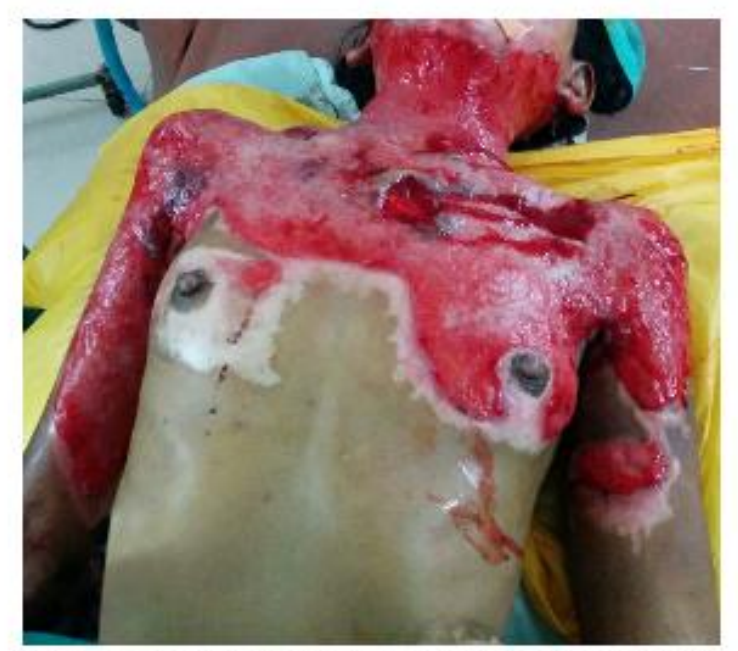

Figure 2: Hemorrhagic bullae seen over the PBRA. 


\section{CASE REPORT}

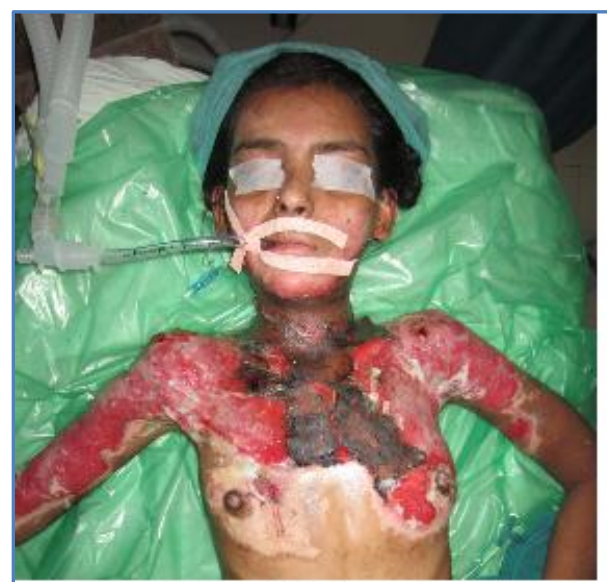

Figure 3: After first stage of

Split Thickness Skin Graft (STSG)

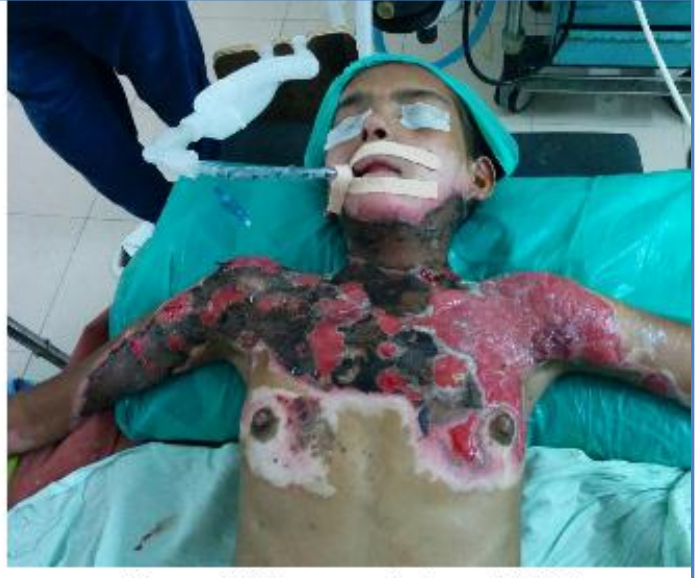

Figure 4: After second stage of STSG
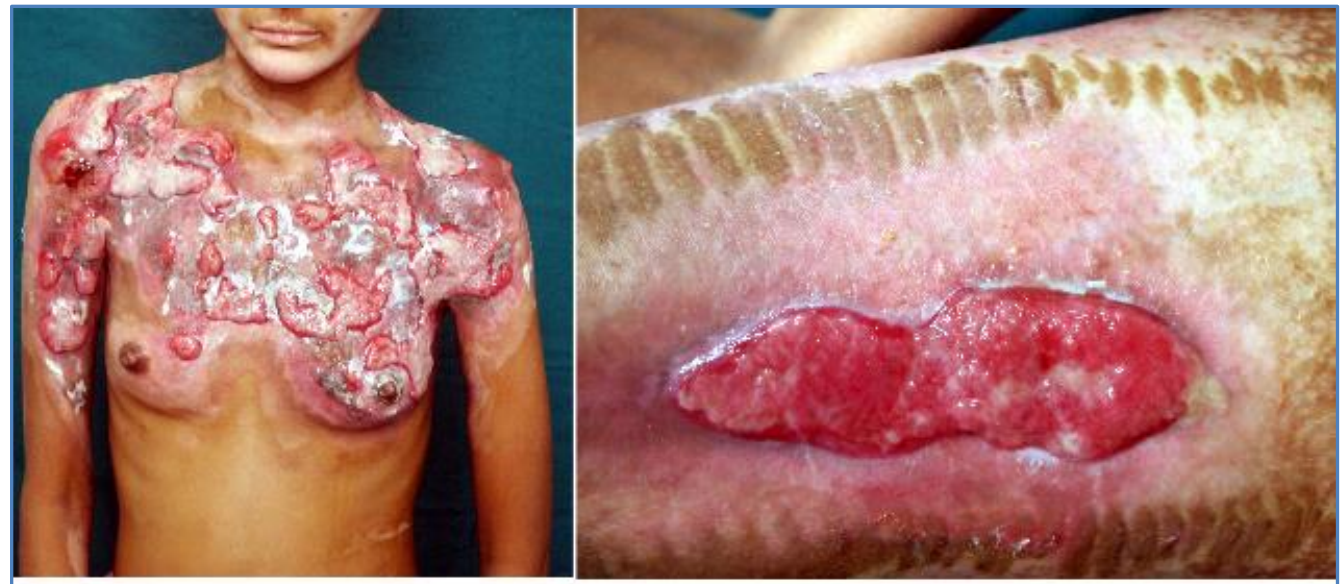

Figure 5: Two months after the third stage of STSG, patient presented with multiple granulating ulcerations over trunk, arms and right thigh.
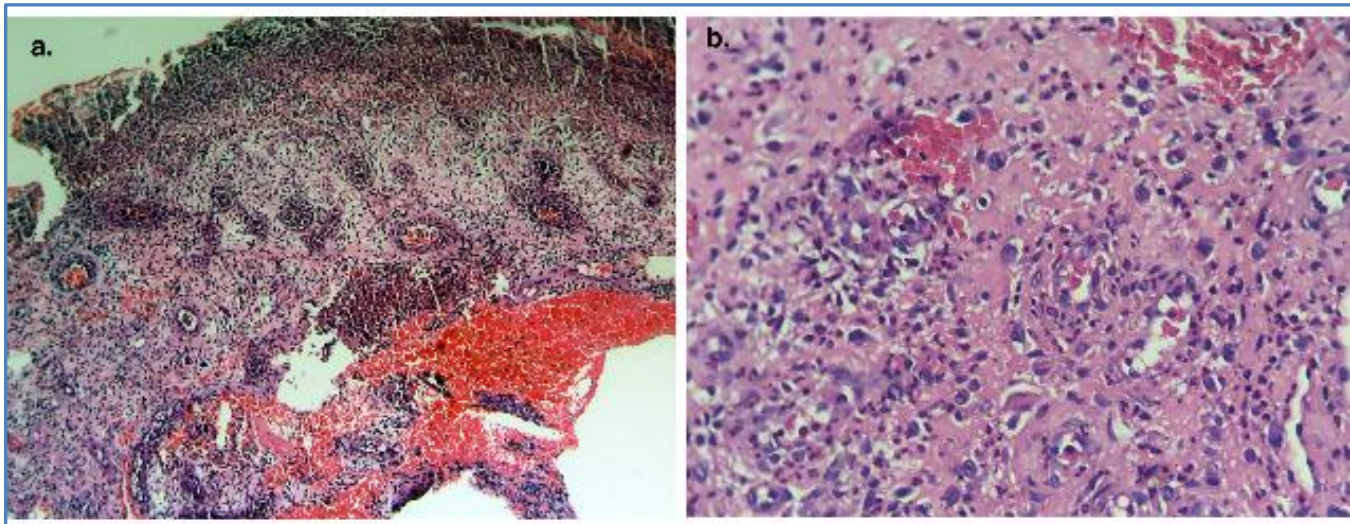

Figure 6: Biopsy shows dense predominantly neutrophilic infiltrate on a background of proliferating granulation tissue involving most of the dermis. ( $H$ \& $E_{;} a: x 40$ \& b: $x 100$ ) 


\section{CASE REPORT}
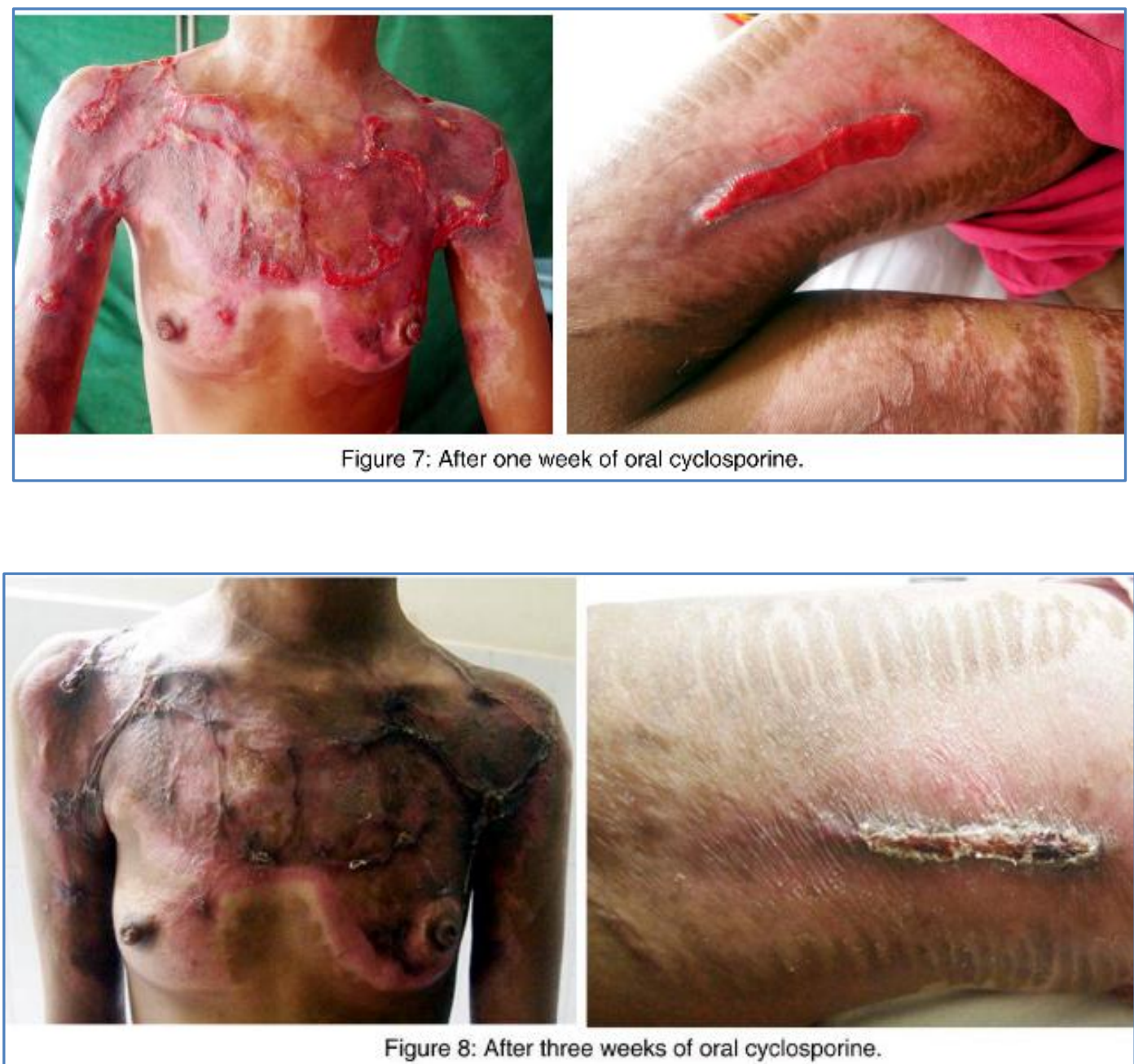

\section{AUTHORS:}

1. Sushma Chowdary Kosaraju

2. Pratap Duggirala

\section{PARTICULARS OF CONTRIBUTORS:}

1. Assistant Professor, Department of Dermatology, NRI Medical College \& General Hospital.

2. Assistant Professor, Department of Plastic Surgery, NRI Medical College \& General Hospital.

\section{FINANCIAL OR OTHER}

COMPETING INTERESTS: None

\section{NAME ADDRESS EMAIL ID OF THE CORRESPONDING AUTHOR:}

Dr. Sushma Chowdary Kosaraju, Assistant Professor,

Department of Dermatology Venereology \& Leprology, NRI Medical College \&

General Hospital, Chinakakani,

Mangalagiri Mandal, Guntur-522 503,

Andhra Pradesh, India.

E-mail: sushma_kosaraju@yahoo.co.in

Date of Submission: 10/04/2015.

Date of Peer Review: 11/04/2015.

Date of Acceptance: 28/04/2015.

Date of Publishing: 07/05/2015. 